\title{
EBSD Investigation of Twinned Copper
}

\author{
Robert E. Goddard, Jonathan Padelford, Ke Han, and Jun Lu
}

National High Magnetic Field Laboratory, Florida State University, Tallahassee, FL 32310

Introduction

Orientation Imaging Microscopy (OIM) is a powerful technique using electron backscatter diffraction (EBSD) to characterize microstructure features with the crystallographic orientation information. Most of the work by OIM has been undertaken on microstructured materials because of the limitation of the resolution of the microscope and the current density. The resolution was affected by both the design of the microscope and the condition of the samples. With a combination of a field emission gun scanning electron microscope (FEGSEM), Focused Ion Beam (FIB), and OIM, we have demonstrated that high resolution can be achieved by OIM in engineered samples.

Procedure and Results

$\mathrm{Cu}$ is one of the commonly used samples in material science research. $\mathrm{Cu}$ has intermediate stackingfault energy and therefore it is difficult to create a high density of twins in commercial $\mathrm{Cu}$. However, twinning is very important in $\mathrm{Cu}$, because it is an important deformation mechanism at cryogenic temperatures and forms $\Sigma 3$ boundaries that has special effects to motions of the dislocations and scattering of the electrons [1]. We have prepared samples with high twin densities with the twin spacing from nanoscale to microscale. Many fabrication variables, e.g. including current density, temperature, substrate strain, time, and chemistry, contribute to the microstructure [2]. To achieve high twin densities, pulse electrodeposition (PED) was used [3]. The final sample thickness is between 0.1 to $0.2 \mathrm{~mm}$. The samples were polished with standard metallographic techniques. The final polish media is composed of 0.05 micron $\mathrm{Al}_{2} \mathrm{O}_{3}$ particles in a $9 \mathrm{pH}$ slurry on a vibratory polisher. The samples were etched by immersion in a solution of $\mathrm{NH}_{4} \mathrm{OH}$ and $\mathrm{H}_{2} \mathrm{O}_{2}$ for 30 seconds and observed first by Confocal Laser Scanning Microscopy (CLSM) (Olympus LEXT 3100) for surface features (Fig. 1).

The samples for CLSM were etched too deep for EBSD and therefore repolished for examination using a Zeiss 1540 Cross Beam FEGSEM. We observed that rapid oxidation resulted in obscured surface features and poor resolution in scanning electron microscope (SEM) images. This also hindered the investigation by EBSD because the patterns intensities were low and the pattern resolution was poor. The oxidation was eliminated by FIB. The FIB generated image is very sensitive to the orientation variations and therefore provides very sharp images of the microstructure from our samples based on crystallographic differences from our samples (Fig. 2). This was verified by EBSD pattern in Fig. 3 using TSL OIM at 20kV. Twins with various spacing were observed by both FIB and EBSD technologies. A couple of areas with the smallest twinning were scanned using ESBD with a step size from 2 to 50 nanometers to determine the size of the twin expression and resolution of the ESBD technique (Fig. 4). To examine the achievable resolution by OIM a twin was selected that had the smallest resolvable width and uniform measurements. A typical image map was obtained with a step size of 5 nanometers. The top of Fig. 4 is an image of the inverse pole figure (IPF) with the image quality (IQ) map below (twin, $60^{\circ}$, boundary highlighted in green). The image demonstrates that the twin boundaries are not straight and show zigzag features in both IPF images and IQ maps. This can be related to the local strain, incoherent steps/boundaries, or 
uncertainty in OIM images with small scan steps. Fig. 5 is a combination map of the IPF and IQ with the misorientation profile (Fig. 6) scan lines across the selected twin. The average of all four scans across the continuous twin identified yields a thickness of 22 nanometers.

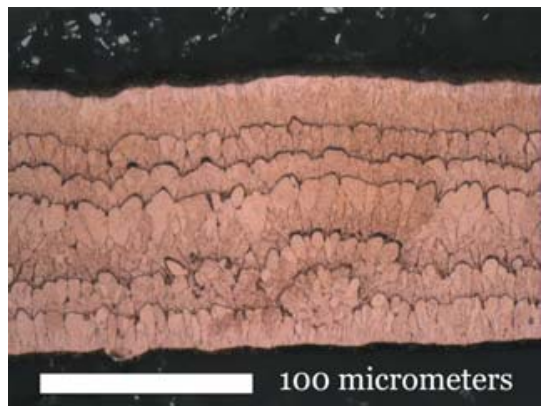

FIG. 1. Etched Longitudinal Cross section

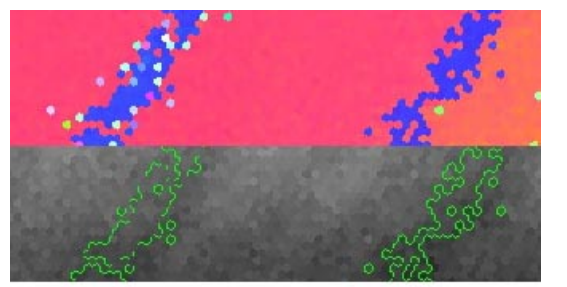
100 nanometers FIG. 4. EBSD Maps

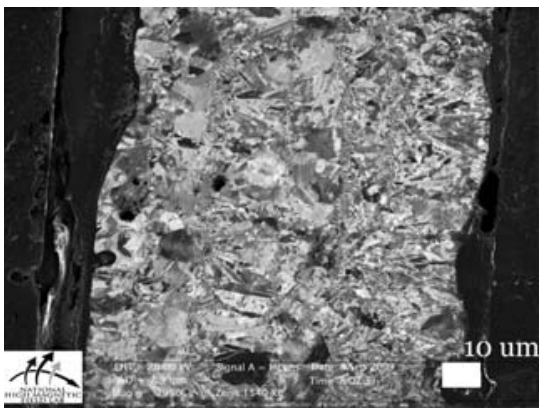

FIG. 2. FIB cleaned

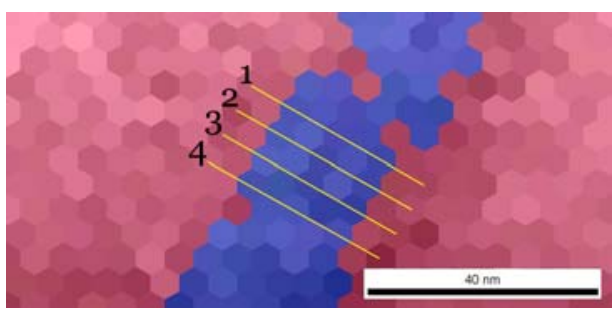

FIG. 5. Combination ESBD Map With scan lines

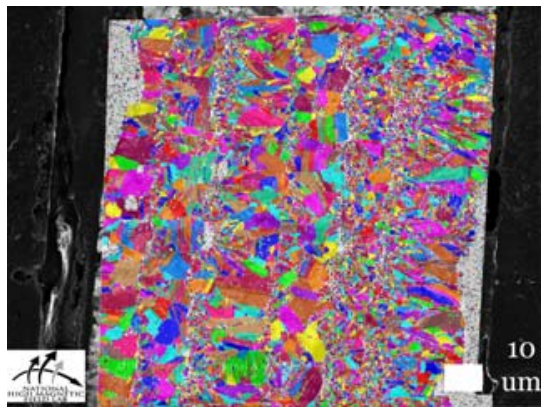

FIG. 3. EBSD Inverse Pole Figure Map

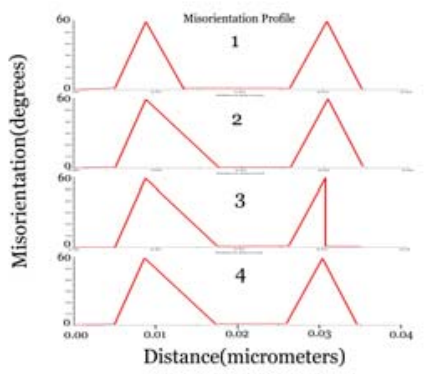

FIG. 6. Misorientation Profile Scan Lines

Discussion

Due to contamination on the sample of the electron beam the sample could not be effectively scanned at a step size below 5 nanometers. The accuracy of the measurement would also be improved by orienting the twin boundary edge-on. The detectable twin limit is probably closer to 20 nanometers with step size of 5 nanometers and twin boundary in arbitrary orientations.

\section{Conclusion}

The increased electron signal by field emission gun and improvement of the lens in FEGSEM provides a unique opportunity to explore the high resolution OIM images. However, sample preparations impose a limitation. By a combination of FIB and OIM in a FEGSEM, both the samples and resolution are improved and fine twins of size down to 20 nanometers can be examined. Therefore, the technology allows one to achieve resolution of 20 nanometers or better.

\section{References}

[1] K. Han, R.P. Walsh, A. Ishmaku, V. Toplosky, and J. D.Embury, "High Strength and High Electrical Conductivity Bulk Cu”, Phil. Mag., 84 (34), 3705-3716, (2004).

[2] Lui Guan-Tai, Chen Delphic, and Kuo Jui-Chao EBSD characterization of twinned copper using pulsed electrodeposition J. Phys. D: Appl. Phys. 42215410

[3] Cui B Z, Han K., XinY., Waryoba D. R., and Mvaruku A. L. Highly textured and twinned Cu films fabricated with pulsed electrodeposition Acta Mater. 55 (2007) 4429-38

This work has been supported by the NSF under award number DMR-9527035. 